\title{
Practical tools to identify short children born small-for-gestational-age eligible for rhGH treatment according to Italian regulation
}

\author{
Gianluca Tornese ${ }^{1 *}$ (D) Flavia Pricci², Maria Chiara Pellegrin ${ }^{1}$, Marika Villa ${ }^{2}$, Daniela Rotondi ${ }^{2}$ Elvira Agazio² and \\ Egidio Barbi i,3
}

\begin{abstract}
Recombinant human growth hormone $(\mathrm{rhGH})$ is an approved and effective treatment for short children born small for gestational age (SGA). Prevalence of children eligible for treatment as SGA is reported to be 1:1800. The latest data from the National Registry of Growth Hormone therapy (RNAOC) showed that the number of children treated with SGA indication is still small (prevalence $0.37 / 100,000$ ) and these children are significantly less reported than those treated for growth hormone deficiency (GHD), although GHD prevalence is 1:4000-1:10,000. This means that many short children born SGA are still not properly identified, and therefore not treated with rhGH, or misdiagnosed as GHD. This article provides some practical tools for the identification of children eligible for rhGH treatment.
\end{abstract}

Keywords: Growth hormone, Small for gestational age, Italy

\section{Background}

To born small for gestational age (SGA) is considered the main reason for short stature in $10 \%$ of short adults.

Children born SGA should be $2 \%$ of the population by definition (-2 SDS correspond to the 2nd percentile). Actually, some population studies have shown different prevalence: $3.1 \%$ in Finland out of 1,390,165 singletons, $5.5 \%$ in Sweden out of 3650 healthy full-term children (37-43 gestational weeks) (of which 1.6\% SGA only for weight, 2.4\% SGA only for length, 1.5\% SGA both for weight and length), $3.5 \%$ in Japan on 27,228 children (3.4\% in term children, $5.5 \%$ in preterm children; $1.2 \%$ SGA only for weight, 1.5\% SGA only for length, $0.8 \%$ SGA both in weight and length) [1].

Catch-up growth is more pronounced during the first 6 months and is usually completed in the first 2 years of life (although preterm born SGA catch-up growth is completed also beyond the first 2 years of life, beyond age 6 years and sometimes in adolescence). Previous

\footnotetext{
* Correspondence: gianluca.tornese@burlo.trieste.it

${ }^{1}$ Institute for Maternal and Child Health IRCCS "Burlo Garofolo", Trieste, Italy Full list of author information is available at the end of the article
}

studies found that $8-12 \%$ of SGA children will have a short stature at 2 years of life and these children have a higher risk of short stature later in life [2,3].

Recombinant human growth hormone (rhGH) is an approved and effective treatment for short children born SGA [4]. Although long-term treatment with rhGH can increase adult height, since SGA children are increasingly recognized as a heterogeneous group in which multiple mechanisms of growth retardation and metabolic disturbances could be causative, it has to be kept in mind - and shared with parents prior to treatment - that the response to rhGH therapy is highly variable and additional studies are needed to identify the responders [5].

SGA children do not need to be deficient in growth hormone $(\mathrm{GH})$ in order to qualify for, or benefit from, rhGH therapy: in fact, the vast majority of SGA children demonstrate GH levels in the normal range, but appear to have low normal circulating IGF-I concentrations. Moreover, SGA children with GH deficiency need higher than replacement dose of rhGH for optimum growth response $(0,035$ $\mathrm{mg} / \mathrm{kg} /$ day), probably because of some IGF-I resistance [6]. Assessment of the GH-IGF-I axis may be required if 
growth velocity is persistently reduced and signs of GH deficiency or hypopituitarism are present.

With the limits of a mechanistic calculus, considering a minimum prevalence of $2 \%$ children born SGA for weight and/or length, the hypothetical prevalence of short children born SGA at the age of 2 years would be $0.24 \%$ (1:417). Only one Japanese study [1] verified the prevalence of children with short stature born SGA by studying an entire cohort of nearly 30,000 children born during a three-year period and re-evaluated at 3 years of life: the prevalence was found to be $0.06 \%(1: 1800)$ (notably greater in preterm births <34 SGA, 0.39\%, 1:256). Although considerably lower than the hypothetical one, this prevalence far exceeds the estimated prevalence of GHD (1:4000-1:10,000).

Although rhGH treatment is not mandatory in every child born SGA without catch-up growth, and parents need to be informed about the variability of response to therapy, we believe that in Italy many short children born SGA are still not properly identified and therefore rhGH treatment is not offered as an option.

Aim of this article is to describe the situation of rhGH prescriptions in short children born SGA in Italy and to provide practical tools for pediatricians to identify children eligible for rhGH treatment according to Italian regulation.

\section{Main text}

Treatment with rhGH has been approved in 2009 by the Italian Medicines Agency (AIFA) for the treatment of children with short stature born SGA and is then reimbursed by the Italian National Health System (Servizio Sanitario Nazionale - SSN) according to the Note \#39 on the use of drugs.

This treatment was approved in 2001 from the American Food and Drug Administration (FDA), in 2003 from the European European Agency for the Evaluation of Medicinal Products (EMEA) and in 2008 from the Ministry of Labor and of Welfare in Japan; eligibility criteria are slightly different from each other.

According to the latest version (2014) of the Note \#39 of AIFA [7], to access treatment with rhGH in individuals born SGA it is necessary to meet all the following criteria (Fig. 1):

- birth weight $\leq-2$ standard deviations score (SDS) $(<$ 3rd percentile) and/or length at birth $\leq-2$ SDS for gestational age according to Bertino charts [8];

- age at the start of GH therapy equal to or greater than 4 years;

- height less than or equal to - 2.5 SDS;

- growth velocity lower than 50th percentile.

In previous regulations, rhGH was provided only for short children born SGA for weight (not for length) and Gagliardi charts had to be used. Bertino charts are the most recent and methodologically more accurate, which differentiate neonatal weights and lengths not only based on sex but also on birth order.

The latest data from the National Registry of Growth Hormone therapy (RNAOC) showed only $311 \mathrm{rhGH}$ prescriptions based on SGA indication versus 3942 based on GHD indication on the overall population of 4584 subjects taking rhGH in childhood in Italy [9]. Moreover, a subanalysis on Registry data, showed that the prevalence of SGA treated patients in 2017 was 0.37 / $100,000(1.79 / 100,000$ when considering the $0-15$ years population) which is extremely lower than expected, with a stable incidence of new treated patient over the last 7 years $(0.42 / 100,000$ per year) (Table 1$)$.

In order not to lose short children born SGA, it would be appropriate for primary care pediatricians to identify their own SGA patients since the first medical encounter and to monitor them over time.

Remarkably, the definition of SGA used in neonatology might sometimes be different from the one reported here (<-2 SDS): the definition of <10th percentile is broadly used to increase sensitivity for hypoglycemic screening and/or other charts may be used. Therefore, SGA definition needs to be re-evaluated.

Pediatricians can use Bertino charts [8] or, more precisely, directly verify the SDS on the website (http://www. inescharts.com) designed by scientific societies (Italian Society of Pediatric Endocrinology and Diabetology - SIEDP,

\begin{tabular}{|l|}
\hline Is the child born SGA by weight and/or length? (see Table 2 and 3) \\
$\downarrow$ \\
Is the child older than 4 years? \\
$\downarrow$ \\
Has the child a height $<-2.5$ SDS? (see Table 4$)$ \\
$\downarrow$ \\
Has the child a growth rate $<50^{\text {th }}$ percentile $(7 \mathrm{~cm} /$ year)? \\
$\downarrow$ \\
Fig. 1 Flow chart to verify the eligibility of a child born SGA for rhGH treatment7
\end{tabular}


Table 1 Prevalence and incidence of rhGH treatment in SGA patients in Italy from 2011 to 2017, according to the National Registry of Growth Hormone therapy data

\begin{tabular}{|c|c|c|c|c|c|c|c|c|c|}
\hline & \multicolumn{7}{|l|}{ Year } & \multirow[b]{2}{*}{ Mean } & \multirow[b]{2}{*}{$\pm \mathrm{DS}$} \\
\hline & 2011 & 2012 & 2013 & 2014 & 2015 & 2016 & 2017 & & \\
\hline \multicolumn{10}{|l|}{ Prevalence } \\
\hline SGA treated patients (n) & 54 & 68 & 65 & 70 & 108 & 140 & 155 & & \\
\hline $\begin{array}{l}\text { Prevalence (per 100,000 } \\
\text { general population/year) }\end{array}$ & 0.37 & 0.47 & 0.44 & 0.28 & 0.22 & 0.27 & 0.37 & 0.35 & 0.09 \\
\hline $\begin{array}{l}\text { Prevalence (per 100,000 } \\
0-15 \text { years population/year) }\end{array}$ & 0.61 & 0.76 & 0.72 & 0.78 & 1.22 & 1.60 & 1.79 & 1.07 & 0.47 \\
\hline \multicolumn{10}{|l|}{ Incidence } \\
\hline New SGA treated patients (n) & 39 & 29 & 30 & 30 & 47 & 49 & 39 & & \\
\hline $\begin{array}{l}\text { Incidence (per 100,000 } \\
\text { general population/year) }\end{array}$ & 0.37 & 0.27 & 0.22 & 0.28 & 0.44 & 0.47 & 0.38 & 0.35 & 0.09 \\
\hline $\begin{array}{l}\text { Incidence (per 100,000 0-15 } \\
\text { years population/year) }\end{array}$ & 0.44 & 0.33 & 0.27 & 0.34 & 0.53 & 0.56 & 0.45 & 0.42 & 0.11 \\
\hline
\end{tabular}

Italian Society of Neonatology - SIN, Italian Society of Medical Statistics and Clinical Epidemiology - SISMEC); however, to facilitate the identification of SGA children, we propose to use two simple tables (Table 2 for weights and Table 3 for lengths): if the weight or the length of a baby is under the data reported in the Tables, it might be SGA therefore an exact calculation is needed through the aforementioned website to confirm it (data in the Tables are referred to a gestational age of $\mathrm{X}$ week +6 days, which is the highest in the gestational week, in order not to lose data, with the exclusion of 42 weeks +3 days which is the maximum included in Bertino charts).

If the child born SGA has a height lower than - 2.5 SDS at 4 years and growth rate is $<50$ th percentile, there is an indication to start treatment with rhGH and the child must be sent to a pediatric endocrinology center (rhGH prescription

Table 2 Weight (in grams) that might be $\leq-2$ SDS according to Bertino charts based on gestational age, sex and birth order calculated based on the formula: $S D S=\frac{\left(\frac{y}{M(t)}\right)^{L(t)}-1}{S(t) \times L(t)}$

\begin{tabular}{|c|c|c|c|c|}
\hline Gestational age & Firstborn males & Not-firstborn males & Firstborn females & Not-firstborn females \\
\hline 23 & 379 & 397 & 355 & 373 \\
\hline 24 & 408 & 429 & 381 & 402 \\
\hline 25 & 449 & 472 & 418 & 441 \\
\hline 26 & 501 & 527 & 466 & 492 \\
\hline 27 & 566 & 597 & 527 & 557 \\
\hline 28 & 647 & 681 & 602 & 636 \\
\hline 29 & 745 & 784 & 693 & 732 \\
\hline 30 & 861 & 906 & 803 & 847 \\
\hline 31 & 999 & 1049 & 933 & 983 \\
\hline 32 & 1160 & 1217 & 1085 & 1142 \\
\hline 33 & 1345 & 1409 & 1260 & 1324 \\
\hline 34 & 1552 & 1625 & 1457 & 1529 \\
\hline 35 & 1779 & 1860 & 1672 & 1753 \\
\hline 36 & 2015 & 2105 & 1896 & 1986 \\
\hline 37 & 2244 & 2344 & 2114 & 2213 \\
\hline 38 & 2448 & 2555 & 2306 & 2414 \\
\hline 39 & 2606 & 2720 & 2456 & 2570 \\
\hline 40 & 2711 & 2830 & 2555 & 2673 \\
\hline 41 & 2767 & 2889 & 2606 & 2729 \\
\hline 42 & 2782 & 2905 & 2619 & 2742 \\
\hline
\end{tabular}


Table 3 Length (in centimeters) that might be $\leq-2$ SDS according to Bertino charts based on gestational age, sex and birth order calculated based on the formula: SDS $=\frac{\left(\frac{y}{M(t)}\right)^{L(t)}-1}{S(t) \times L(t)}$

\begin{tabular}{|c|c|c|c|c|}
\hline Gestational age & Firstborn males & Not-firstborn males & Firstborn females & Not-firstborn females \\
\hline 23 & 26.3 & 26.6 & 25.8 & 26.1 \\
\hline 24 & 27.4 & 27.7 & 26.9 & 27.2 \\
\hline 25 & 28.6 & 28.9 & 28.1 & 28.3 \\
\hline 26 & 29.8 & 30.1 & 29.3 & 29.5 \\
\hline 27 & 31.1 & 31.4 & 30.5 & 30.8 \\
\hline 28 & 32.4 & 32.7 & 31.8 & 32.1 \\
\hline 29 & 33.7 & 34.1 & 33.1 & 33.4 \\
\hline 30 & 35.1 & 35.5 & 34.5 & 34.8 \\
\hline 31 & 36.5 & 36.9 & 35.9 & 36.2 \\
\hline 32 & 38.0 & 38.4 & 37.3 & 37.7 \\
\hline 33 & 39.5 & 39.8 & 38.7 & 39.1 \\
\hline 34 & 40.9 & 41.3 & 40.1 & 40.5 \\
\hline 35 & 42.3 & 42.7 & 41.5 & 41.9 \\
\hline 36 & 43.6 & 44.0 & 42.8 & 43.2 \\
\hline 37 & 44.8 & 45.2 & 44.0 & 44.4 \\
\hline 38 & 45.8 & 46.3 & 45.0 & 45.4 \\
\hline 39 & 46.7 & 47.1 & 45.8 & 46.3 \\
\hline 40 & 47.3 & 47.8 & 46.4 & 46.9 \\
\hline 41 & 47.8 & 48.2 & 46.9 & 47.4 \\
\hline 42 & 48.0 & 48.4 & 47.1 & 47.5 \\
\hline
\end{tabular}

is subject to a therapeutic plan, signed by specialized centers which are identified by Regions and Autonomous Provinces). To calculate height SDS, pediatricians can use the Growth Calculator distributed by SIEDP on the website http://www.weboriented.it/gh4/; however, to facilitate the identification of short children we propose to use Table 4 . For practical purposes, it may be useful to remind that a growth rate of $7 \mathrm{~cm}$ in the previous year is equal to the 50th percentile according to Tanner charts.

\section{Conclusions}

In conclusion, data suggest that SGA children with short stature are under recognized and frequently miss an opportunity of treatment in Italy. We strongly encourage the use of these simple and time-sparing tables for the identification and appropriate care of SGA children.

Table 4 Height (in centimeters) equal to -2.5 SDS at 4 years of age (Cacciari charts [10] for Italian children or WHO charts [11] for other backgrounds)

\begin{tabular}{lll}
\hline & Males & Females \\
\hline Cacciari charts & 91.97 & 91.55 \\
WHO $<5$ years charts & 92.85 & 92 \\
\hline
\end{tabular}

\section{Abbreviations}

GHD: Growth hormone deficiency; rhGH: recombinant human growth hormone; RNAOC: National Registry of Growth Hormone therapy; SGA: Small for gestational age

\section{Acknowledgments \\ Not applicable.}

\section{Authors' contributions}

GT concepted and wrote the work; FP, MV, DR, EA performed the analysis on National Registry of Growth Hormone therapy data, MP helped in the acquisition of data for Tables 2 and 3, EB have drafted the work and substantively revised it. All authors have approved the submitted version.

\section{Funding}

None.

\section{Availability of data and materials}

Data sharing is not applicable to this article as no datasets were generated during the current study.

Ethics approval and consent to participate

Not needed.

\section{Consent for publication}

Not needed.

\section{Competing interests}

The authors declare that they have no competing interests.

\section{Author details}

'Institute for Maternal and Child Health IRCCS "Burlo Garofolo", Trieste, Italy. ${ }^{2}$ Department Cardiovascular, endocrine-metabolic disease and aging, Istituto Superiore di Sanità, Rome, Italy. ${ }^{3}$ University of Trieste, Trieste, Italy. 
Received: 18 July 2019 Accepted: 10 September 2019

Published online: 21 October 2019

References

1. Fujita K, Nagasaka M, Iwatani S, et al. Prevalence of small for gestational age (SGA) and short stature in children born SGA who qualify for growth hormone treatment at 3 years of age: population-based study. Pediatr Int. 2016;58:372-6.

2. Albertsson-Wikland K, Karlberg J. Postnatal growth of children born small for gestational age. Acta Paediatr Suppl. 1997;423:193-5.

3. Leger J, Limoni C, Czernichow P, et al. Prediction of the outcome of growth at 2 years of age in neonates with intra-uterine growth retardation. Early Hum Dev. 1997;48:211-23.

4. Lee PA, Chernausek SD, Hokken-Koelega AC, Czernichow P. Internationa Small for Gestational Age Advisory Board. International Small for Gestational Age Advisory Board consensus development conference statement: management of short children born small for gestational age, April 24October 1, 2001. Pediatrics. 2003;111:1253-61.

5. Maiorana A, Cianfarani S. Impact of TH therapy on adult height of children born small for gestational age. Pediatrics. 2009;124:e519-31.

6. de Zegher F, Francois I, van Helvoirt M, et al. Growth hormone treatment of shor children born small for gestational age. Trends Endocrinol Metab. 1998;9(6):233-7.

7. Italia. Determinazione dell'Agenzia Italiana del Farmaco 19 giugno 2014. Modifica alla Nota AIFA 39. Gazzetta Ufficiale. Serie Generale n. 154 del 5 luglio 2014.

8. Bertino E, Spada E, Occhi L, et al. Neonatal anthropometric charts: the Italian neonatal study compared with other European studies. J Pediatr Gastroenterol Nutr. 2010;51:353-61.

9. Pricci F, Villa M, Maccari F, et al. The Italian registry of $\mathrm{GH}$ treatment: electronic clinical report form (e-CRF) and web-based platform for the national database of GH prescriptions. J Endocrinol Investig. 2018;42:769-77 [Epub ahead of print].

10. Cacciari E, Milani S, Balsamo A, et al. Italian cross-sectional growth charts for height, weight and BMI (2 to 20 yr). J Endocrinol Investig. 2006:29:581-93.

11. WHO Multicentre Growth Reference Study Group. WHO child growth standards based on length/height, weight and age. Acta Paediatr. 2006:450(Suppl 450):76-85.

\section{Publisher's Note}

Springer Nature remains neutral with regard to jurisdictional claims in published maps and institutional affiliations.

Ready to submit your research? Choose BMC and benefit from:

- fast, convenient online submission

- thorough peer review by experienced researchers in your field

- rapid publication on acceptance

- support for research data, including large and complex data types

- gold Open Access which fosters wider collaboration and increased citations

- maximum visibility for your research: over $100 \mathrm{M}$ website views per year

At $\mathrm{BMC}$, research is always in progress.

Learn more biomedcentral.com/submissions 